\title{
Management der radiogenen Zystitis
}

_ In Folge einer Strahlentherapie bei urologischen und gynäkologischen Tumoren können Patienten eine radiogene Zystitis entwickeln. Eine Heilung ist bislang nicht möglich, aber es gibt Strategien für das Management einer solchen Blasenentzündung.

Ausgelöst durch die ionisierenden Strahlen wird das Blasengewebe geschädigt. Die Strahlung löst einen Teufelskreis aus, in dem sich Fibrosierung und Ischämie gegenseitig und progredient verschlimmern. Fibrosierung und Ischämie führen dazu, dass das Urothel seine beiden wichtigsten Funktionen - Flexibilität und Barrierewirkung - nur noch unzureichend wahrnehmen kann. Über einen Zeitraum von sechs Wochen bis $14 \mathrm{Jah}$ re nach der Strahlentherapie kann sich somit eine radiogene Zystitis entwickeln.

Eine effektive Therapiemöglichkeit ist die intravesikale Instillation von 0,2\%-igem Chondroitinsulfat (Gepan instill ${ }^{\oplus}$ ). Diese beruht auf der Beobachtung, dass Chondroitinsulfat ein essenzieller Bestandteil der Glykosaminoglykan-Schicht (GAG) ist. Durch die Applikation von Chondroitinsulfat kann das Defizit ausgeglichen werden und die Defekte in der GAG-Schicht können behoben werden. Der klinische Erfolg einer intravesikalen Instillationstherapie mit Chondroitinsulfat 0,2\% wurde in verschiedenen Studien bei Patienten mit interstitieller Zystitis, OAB-Syndrom und radiogener Zystitis dokumentiert. Eine internationale Beobachtungsstudie mit Patienten, die an chronischer Blasenentzündung unterschiedlicher Ursache litten, hat die gute Wirksamkeit und Verträglichkeit der GAG-Ersatztherapie belegt [Nordling J et al. Drug Research 2008; 58: 328-35]. Von den insgesamt 286 Studienteilnehmern litten $5 \%$ an einer radiogenen Zystitis. Die Patienten erhielten in den ersten 4-6 Wochen Instillationen von $40 \mathrm{ml}$ Chondroitinsulfat im wöchentlichen Abstand und danach eine Erhaltungstherapie mit monatlichen Instillationen. Die Studie ergab, dass die Behandlung mit intravesikulärer 0,2\%-iger Chondroitinsulfat-Lösung die Symptome um 25-50\% verbesserte.

Nach Informationen von

Pohl-Boskamp, Hohenlockstedt
Relaunch von prostata-info.de

Mit dem Relaunch der Website www. prostata-info.de stellt GlaxoSmithKline ein vollständig überarbeitetes OnlinePortal rund um die Prostata und die Benigne Prostatahyperplasie (BPH) zur Verfügung. Auf prostata-info.de wird Männern die Möglichkeit geboten, sich umfassend über die Themen Prostata und BPH zu informieren. Auf der Startseite von prostata-info.de können Interessierte den IPSS-Fragebogen (Internationaler ProstataSymptomen-Score) ausfüllen. Bereits nach wenigen Fragen erhält der Nutzer das Ergebnis des Prostata-Selbsttests und entsprechenden Rat, wie das weitere Vorgehen aussehen könnte. Auch Ängste und die Scheu vor dem Besuch beim Urologen sollen durch die angebotenen Inhalte abgebaut werden. Neben dem Online-IPSS-Test bietet die Website zum Beispiel ein Glossar der Fachbegriffe rund um Prostata und BPH, eine Link-Sammlung zu Themen wie Selbsthilfe oder Therapie sowie Tipps zu Gesundheitsvorsorge, Ernährung und Sport.

Nach Informationen von

GlaxoSmithKline, München

\section{Belatacept zugelassen}

Die Europäische Kommission hat die Zulassung für Nulojix ${ }^{\circledast}$ (Belatacept) erteilt. Belatacept ist in Kombination mit Kortikosteroiden und einer Mycophenolsäure indiziert für die Prophylaxe einer Transplantatabstoßung bei Erwachsenen, die eine Nierentransplantation erhalten haben. Für die Induktionstherapie wird empfohlen, diesem Belatacept-basierten Regime einen Interleukin (IL)-2-Rezeptorantagonisten hinzuzufügen. Belatacept ist das erste Molekül mit einem neuen Wirkmechanismus bei Nierentransplantationen seit zehn Jahren. Durch die selektive Wirkweise im Immunsystem bei der Prophylaxe der Transplantatabstoßung hilft Belatacept, die Nierenfunktion aufrecht zu erhalten. Dieses Kriterium wird immer mehr als eine der Schlüsselkomponenten bei den Langzeitergebnissen angesehen, die sowohl das Transplantat- als auch das Patientenüberleben beeinflussen.

Nach Informationen von Bristol-Myers Squibb, München
Nach Information von

Pfizer, Berlin rapie mit Sunitinib (Sutent ${ }^{\circledast}$ ) oder mit Zytokinen durchgeführt wurde. Das Sicherheitsprofil von Axitinib in der AXIS-Studie war insgesamt nisse aller Grade wurden unter Axitinib häutonie $(40 \%)$, Fatigue (39\%), Dysphonie (31\%) Eine weitere Auswertung der AXIS-Studie evaluierte mithilfe von Patientenfragebögen mCC-Patienten, die Axitinib erhalten hatten, fische Lebensqualität wie die mit Sorafenib das Auftreten einerverschlechterten qualität durch zuvor definierte Parameter wie Krankheitsprogress und Tod verzögert

uropäische Arzneimittelbehörde EMA Culassungsantrag für Axitinib be Patienten mit fortgeschrittenem Nierenzellkarzinom nach Progress unter einer systemischen Therapie angenommen.

$(19,4 \%$ vs. $9,4 \%, p=0,0001)$. Wie die An weiter zeigte, war der Vorteil bezüglich des PFS unabhängig davon, ob die ErstlinientheURO-NEWS 2011:15(9) 